\title{
Harnessing biomaterials for therapeutic strategies against COVID-19
}

\author{
Thibault Colombani $^{1} \cdot$ Zachary J. Rogers $^{1} \cdot$ Loek J. Eggermont $^{1}$ - Sidi A. Bencherif ${ }^{1,2,3,4}$ (D)
}

Received: 8 December 2020 / Accepted: 24 January 2021 / Published online: 6 April 2021

(C) Qatar University and Springer Nature Switzerland AG 2021

\begin{abstract}
With the emergence of the coronavirus disease 2019 (COVID-19), the world is experiencing a profound human health crisis. The number of infections and deaths due to the severe acute respiratory syndrome coronavirus 2 (SARS-CoV-2) continues to increase every minute, pinpointing major shortcomings in our ability to prevent viral outbreaks. Although several COVID-19 vaccines have been recently approved for emergency use, therapeutic options remain limited, and their long-term potency has yet to be validated. Biomaterials science has a pivotal role to play in pushing the boundaries of emerging technologies for antiviral research and treatment. In this perspective, we discuss how biomaterials can be harnessed to develop accurate COVID-19 infection models, enhance antiviral drug delivery, foster new antiviral strategies, and boost vaccine efficacy. These efforts will not only contribute to stop or mitigate the current pandemic but will also provide unorthodox platforms to understand, prevent, and protect us from future viral outbreaks.
\end{abstract}

\section{Introduction}

Since December 2019, the world is facing an unprecedented pandemic caused by a novel coronavirus, the severe acute respiratory syndrome coronavirus 2 (SARS-CoV-2). As of December 2020, more than 63 million patients have been diagnosed worldwide with the novel coronavirus disease 2019 (COVID-19), and the death toll exceeds 1.47 million [1]. Although the current outbreak had long been predicted $[2,3]$, it has pushed and continues to push the healthcare system in many places to its limit with no indications of slowing down. Despite tremendous efforts, only remdesivir, a broad-spectrum antiviral medication, has been approved by the FDA for the treatment of COVID-19. However, the efficacy of remdesivir is in question following a report from the

Sidi A. Bencherif

s.bencherif@northeastern.edu

1 Department of Chemical Engineering, Northeastern University, Boston, MA 02115, USA

2 Department of Bioengineering, Northeastern University, Boston, MA 02115, USA

3 Harvard John A. Paulson School of Engineering and Applied Sciences, Harvard University, Cambridge, MA 02138, USA

4 Biomechanics and Bioengineering (BMBI), UTC CNRS UMR 7338, University of Technology of Compiègne, Sorbonne University, 60203 Compiègne, France
World Health Organization's "solidarity" clinical trial, which showed little to no effect in improving patient survival or shortening hospitalization time [4]. Without effective antiviral drugs or other treatments that halt or alleviate continued viral infections, patients must rely on their own immune system. The situation is critical, and only coordinated collaborative efforts and coalitions among leaders across various interdisciplinarity fields at a global level will give us the tools to win the battle against this common enemy.

Biomaterials science has played a central role in a number of exciting medical advances that include the discovery of new therapeutic strategies [5]. Biomaterials are derived from natural or synthetic materials that have been engineered to interact with biological systems for therapeutic or diagnostic purposes. For instance, they provide a unique means to bioengineer complex microenvironments to better recapitulate and study human diseases [6,7]. Such microenvironments can also be used as high-throughput screening platforms to test the effectiveness of new and emerging therapeutics. In addition, biomaterials can be used as effective platforms for drug delivery and vaccination, and can be designed to exhibit antiviral activities [8]. The types of biomaterials that are of particular relevance include hydrogels, cryogels, and nanoparticles (NPs). Hydrogels are hydrophilic, three-dimensional (3D) crosslinked polymers [9-14]. Due to their high water content, hydrogels tend to be biocompatible and have been extensively used for several biomedical applications, such as tissue engineering and drug delivery [15]. Cryogels, a

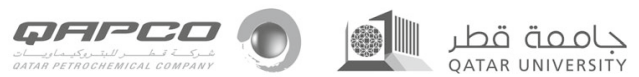


subclass of hydrogels fabricated at subzero temperatures, exhibit unique properties, including a macroporous network, shape memory, and syringe injectability, and have been used for several bio-related applications, including bioseparation, tissue engineering, and immunotherapy [16-23]. NPs, such as liposomes, are nanoscale biomaterials that have been major players in modern medicine; their clinical use ranges from contrast agents to nanocarriers for drug and gene delivery [24, 25].

The purpose of this perspective is to discuss how biomaterials can be harnessed to design advanced COVID-19 infection models, improve current drug delivery methods, develop new antiviral strategies, and reinforce vaccine efficiency. As a platform technology, biomaterials will not only be a game changer in the fight against our ongoing viral pandemic but also help respond to future infectious disease outbreaks.

\section{Antiviral therapies}

Antiviral therapies were attempted as a first-line treatment to alleviate severe COVID-19 at the beginning of the pandemic. Direct-acting antivirals (DAAs) are preferred over indirectacting antivirals, because DAAs are considered safer, despite their susceptibility to becoming ineffective as a result of viral mutations [26]. Due to the immediate and urgent need presented by the pandemic and the lack of existing antiviral drugs targeting SARS-CoV-2, repurposing of available antiviral drugs has been favored. Unlike the development of new antiviral drugs, drug repurposing has potentially shorter study timelines and lower costs in reaching clinical practice for the new application. Several non-specific antiviral agents, such as remdesivir, hydroxychloroquine (HCQ), and the combination of lopinavir (LPV) and ritonavir (RTV), as well as specific inhibitors of viral proteins, such as viral protease inhibitors and viral replication inhibitors, have been investigated in clinical trials. In spite of impressive efforts, the results have been disappointing, and little progress has been made towards the discovery of effective SARS-CoV-2 therapeutics. HCQ, an antimalarial medication that was recently in the spotlight, has demonstrated no clear therapeutic benefit to hospitalized patients $[27,28]$. Similarly, broad-spectrum antiviral molecules, such as remdesivir and LPV/RTV, have shown little to no effect on reducing the hospitalization rate of COVID19 patients [4]. The only treatments that have demonstrated some efficacy in reducing mortality from severe COVID-19 are anti-inflammatory medicines such as corticosteroids, leaving those infected with no effective options for limiting viral spread and disease progression [29]. This shortcoming highlights the crucial need for the design of more accurate drug screening models, new drug delivery platforms, and innovative antiviral strategies. Fortunately, the field of biomaterials has played a key role in the development of life-saving solutions in response to viral diseases [30,31], spanning from virus-deactivating surface coatings [25] to treatment strategies and vaccines $[32,33]$. Biomaterials are poised to meet these challenges and help us get through this crisis and prevent future ones (Fig. 1).

\subsection{Drug screening models}

The lack of physiologically relevant in vitro models to understand both the host immune response against SARS-CoV-2 and the mechanisms of replication and transmission of this virus severely hampers the identification of therapeutic targets [37]. The current gold standard for antiviral therapeutic screening consists of Vero cells, which are kidney epithelial cells isolated from an African green monkey and are grown as a two-dimensional (2D) monolayer [38, 39]. Vero cells are highly susceptible to viral infection, likely because they are genetically deficient in the type I interferon gene cluster [40]. However, this lack of type I interferon has implications for their use as an antiviral screening system. Not only is interferon signaling the first line of defense against viral infections, but it is also an important regulator of angiotensin-converting enzyme 2 (ACE2) - the identified receptor involved in SARSCoV-2 infection [41]. The ability to translate discoveries with Vero cells into the clinic has been challenging for COVID-19. For example, remdesivir, chloroquine, and HCQ inhibit viral infection in Vero cells $[42,43]$, but clinical trials have shown little to no benefit in patients [4].

A limitation of 2D cell culture as a platform for disease modeling and drug screening comes from an inability to recapitulate native tissue complexity and the biophysical properties that contribute to host infection [44-46]. However, biomaterials, such as biologically and chemically defined scaffolds, can support the formation and maturation of relevant human tissues in 3D [47]. For instance, human small airway epithelial cells cultured on chitosan-collagen scaffolds recreate an air-liquid interface and model the human lower airway epithelium. This engineering approach was used to accurately evaluate cytokine production following viral infection [48]. Similarly, in a different strategy, collagen-hyaluronate scaffolds were leveraged to support an in vitro tracheobronchial epithelial co-culture model in 3D by co-culturing human epithelial cells and human lung fibroblasts [49].

Organoids derived from stem cells may also be used to model SARS-CoV-2 infection by recapitulating the complexity and spatial heterogeneity of human organs at a macroscale level [50-52]. For example, studies with human intestinal organoids revealed that SARS-CoV-2 infects and replicates efficiently in the human intestinal tract [53]. Furthermore, vascularized kidney organoids have been crucial for demonstrating the capacity of soluble human ACE2 to inhibit SARSCoV-2 infection in a preclinical system [54]. However, organoid formation often relies on the use of Matrigel, a 
a Synthetic Scaffolds to Support Organoid Formation

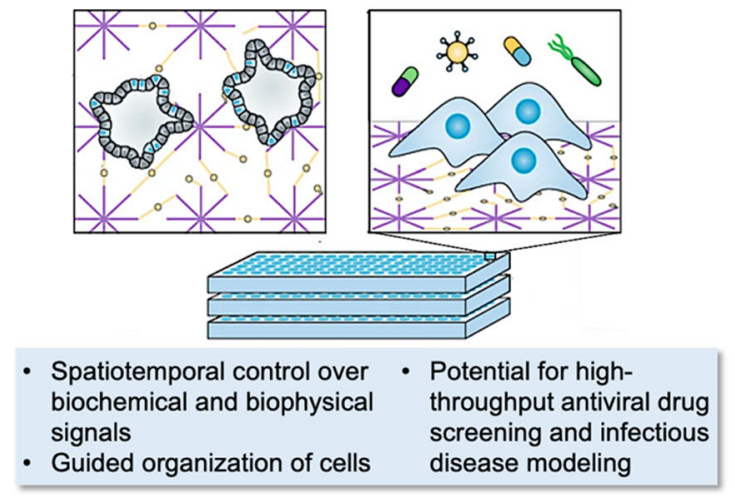

b Decoy Nanoparticles (Nanodecoys) Protect against SARS-CoV-2

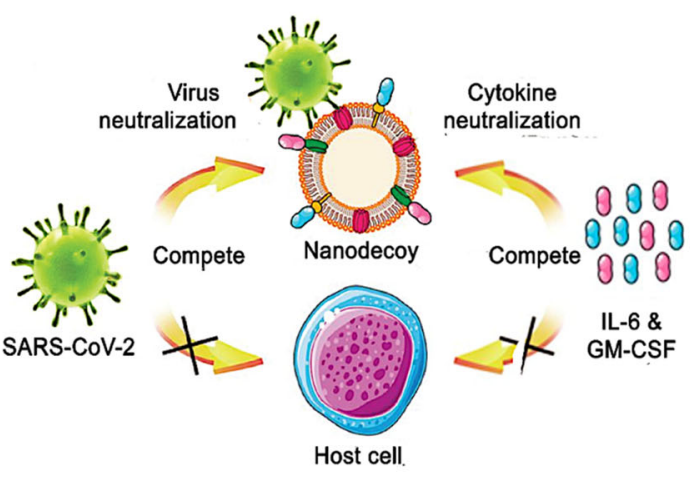

\section{Nanoparticle (NP)-based System for Antiviral Drug Delivery}

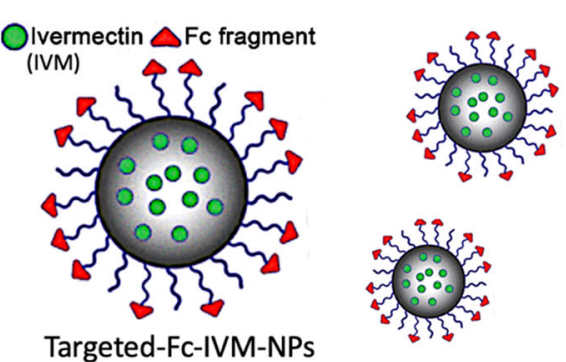

Fig. 1 Leveraging biomaterials to improve antiviral therapeutics. a Schematic of synthetic scaffolds with chemically defined features and tunable biological, mechanical, and physical properties to support organoid formation and potentially develop more accurate viral infection models (reproduced and modified with permission from Aisenbrey et al. [34]). b Schematic highlighting engineered nanodecoys displaying angiotensin-converting enzyme 2 (ACE2) and cytokine receptors against COVID-19. Cellular membrane nanovesicles from genetically edited 293T cells expressing ACE2 and THP-1 cells (human monocytic cell line) were fused and used to protect host cells by neutralizing

xenogeneic hydrogel system with several limitations, such as batch-to-batch variability and inconsistent biophysical and biochemical properties. Engineered biomaterials can provide an alternative to Matrigel with improved and more reliable properties [34]. For instance, bioengineered scaffolds designed to create a native tissue-like extracellular matrix (ECM) support organoid maturation and function, and provide a microenvironment for the co-culture of stromal and immune cells [47].

Organ-on-chip technologies could also be leveraged to model human responses to SARS-CoV-2 infection and narrow the gap between in vitro disease models and human pathophysiology. These biochips can recreate tissue-tissue interfaces, fluid flows, mechanical cues, and organ-level physiology. For example, small airway-on-a-chips containing primary human airway bronchiolar epithelial cells that express high levels of ACE2 and transmembrane protease serine 2 (TMPRSS2) have been used to identify amodiaquine and toremifene as effective SARS-CoV-2 inhibitors $[55,56]$. Furthermore, a

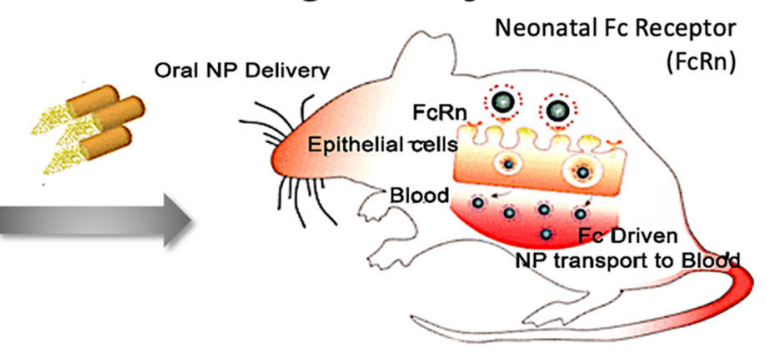

SARS-CoV-2 and delivering inflammatory cytokines, such as IL-6 and GM-CSF (reproduced and modified with permission from Rao et al. [35]). c Illustration describing the development and optimization of NPs loaded with ivermectin (IVM) for oral administration of antiviral drugs. Poly(lactide-co-glycolide)- $b$-polyethylene glycol (PLGA- $b$-PEG)-based NPs were coated with the antibody Fc fragment to target epithelial cells and used to carry poorly water-soluble drugs and increase their half-life in circulation while minimizing toxicity (reproduced and modified with permission from Surnar et al. [36])

microengineered gut-on-chip system mirroring intestinal barrier injury and the human response to viral infection was reconstituted through the co-culture of human intestinal epithelial, mucin-secreting, and vascular endothelial cells. This approach has been central in demonstrating the alteration of the vascular endothelium by SARS-CoV-2, which may explain diarrhea and hemorrhagic colitis symptoms in COVID-19 patients [57]. However, the organ-on-chip approach still fails to precisely recapitulate native tissue microenvironments and achieve the required biological relevance. Integrating biodegradable and biomimetic scaffolds such as hydrogels or cryogels made of bioactive polymers (e.g., hyaluronic acid, gelatin, fibrin) within these microfluidic cell culture biochips could emulate the native extracellular microenvironment and, as a result, support and guide microvascularization [58].

In light of these new findings, a robust model to study SARS-CoV-2 infection mechanisms and effectively screen antiviral drugs should encompass the following characteristics: (i) integrate tissue-specific human cells; (ii) co-culture 
relevant cells, including immune cells, stromal cells, and epithelial cells; (iii) recapitulate a close-to-native tissue or organ architecture; and (iv) display de novo ECM deposited by cells prior to viral contamination (Fig. 1a). Such models can be engineered with state-of-the-art biomaterials such as sophisticated hydrogel systems $[59,60]$.

\subsection{Drug delivery systems}

Antiviral drug candidates often exhibit short half-lives and limited solubility, leading to low bioavailability and suboptimal therapeutic effect [25]. Furthermore, many antiviral molecules have unwanted off-target-based toxicologic effects [61]. As a result, most antivirals are not suited for systemic administration, hampering their clinical potential. To overcome these challenges, biomaterial-based delivery systems could be harnessed not only to reduce drug dose and stabilize antiviral agents but also to provide better tissue targeting, alleviate systemic exposure, and limit off-target adverse effects. Properties of biomaterials such as physicochemical characteristics, stimuli responsiveness, size, and geometry can be adjusted to enhance biocompatibility and biostability, as well as to control and target drug delivery [62, 63]. Ultimately, biomaterials can provide a transformative solution to bolster safer antiviral drug candidates. For instance, phosphatidylcholine-based liposomes [64] and poly(lactide-coglycolide)-polyethylene glycol block copolymer (PLGA- $b$-PEG) NPs [36] have been used as drug delivery carriers for ivermectin, a promising antiviral therapeutic that reduces SARS-CoV-2 replication in Vero cells [65]. When incorporated into phosphatidylcholine-based liposomes, ivermectin exhibited low in vitro cytotoxicity to uninfected cells while demonstrating antiviral activity in infected cells [66]. In a different approach, ivermectin formulated with PLGA-b-PEG NPs was orally administered, resulting in long-lasting drug bioavailability and pharmacokinetics in mice [36]. Similarly, phosphatidylcholine-cholesterol lipid NPs co-encapsulating LPV and RTV with tenofovir improved anti-HIV drug persistence in macaques [67]. While none of these strategies has been applied to SARS-CoV-2, prior studies suggest that such approaches have great potential. Furthermore, liposomes and NPs could deliver multiple antiviral agents simultaneously or sequentially for a synergistic therapeutic effect (Fig. $1 b)$.

When considered as drug delivery systems, biomaterials exhibit a high degree of flexibility in terms of their mode of administration. They can be introduced into the body by oral administration, surgical implantation, injection, or inhalation. Inhalation would result in local pulmonary drug delivery while minimizing adverse side effects [68]. For instance, when tested in rats, intratracheal instillation of dipalmitoylphosphatidylcholine/ cholesterol liposomes encapsulating HCQ resulted in increased drug half-life and local accumulation into lung tissues with minimal off-target toxicity [69]. These biomaterials can also be used to deliver dexamethasone, a steroid used to treat patients critically ill with COVID-19 [56]. The pulmonary delivery of dexamethasone with liposomes could potentially outperform standard dexamethasone administration by targeting hyperactive immune cells in acute phase of COVID-19 and by mediating enhanced anti-fibrotic activity during the recovery phase [70].

Biomaterials can also improve a number of therapeutic strategies and have recently gained attention for reducing mortality in COVID-19 patients. For instance, interleukin 6 (IL-6) inhibitors have taken center stage amid the pandemic, given the central role of IL- 6 in the cytokine storm phase of the disease [71]. IL-6 inhibitors (e.g., sarilumab, tocilizumab) have been used to alleviate this life-threatening proinflammatory response in critically ill patients with generally promising results [71]. Here, there is an opportunity for biomaterials to be leveraged for the controlled delivery of these drugs and to potentially overcome common complications of systemic administration, while mitigating disease severity [72]. Similarly, given the encouraging data from a severe COVID-19 patient successfully treated with human recombinant soluble angiotensin-converting enzyme 2 (hrsACE2), biomaterials should be considered to further consolidate its therapeutic benefit and safety [73, 74].

\subsection{Cellular nanovesicles}

Biomaterial-centered strategies, such as cell-mimicking NPs, are also a promising avenue to fight COVID-19. Cellmimicking NPs emulate the characteristics of living cells and often contain components derived from cells. These particles act as nanodecoys to trap and sequester SARS-CoV-2 virus or as nanosponges to absorb and neutralize proinflammatory cytokines to alleviate cytokine storms in patients with severe COVID-19. For instance, cellular nanovesicles (CN) fabricated with a PLGA core and a membrane from either human lung epithelial type II cells (epithelial-CN) or human macrophages (macrophage- $\mathrm{CN}$ ) showed high neutralizing activity against SARS-CoV-2, preventing viral infection of Vero cells [75]. This mechanism was mediated via binding to the host viral entry receptors ACE2, TMPRSS2, and dipeptidyl peptidase IV (DPP4) located on the surface of the epithelial$\mathrm{CN}$, as well as ACE2, C-type lectin domain family 10 (CLEC10), and CD147 receptors found on the surface of the macrophage-CN. Similarly, anti-inflammatory NPs were engineered with membranes derived from murine macrophages containing the lipopolysaccharide (LPS)-binding receptors CD14 and toll-like receptor 4 (TLR4). Additionally, the NPs displayed cytokine-binding receptors CD126 and CD130 for interleukin 6 (IL-6), CD120a and CD120b for tumor necrosis factor alpha (TNF- $\alpha$ ), and CD119 for interferon gamma (IFN- $\gamma$ ). In a murine infection model of Gramnegative bacterial sepsis, these NPs successfully reduced levels of proinflammatory cytokines, specifically IL-6, TNF- $\alpha$, and IFN- $\gamma[76]$. 
Other NPs combine the functions of nanodecoys and nanosponges. These $\mathrm{CNs}$ were fabricated by mixing materials from human epithelial kidney cells expressing ACE2 and human monocytes. The resulting NPs displayed ACE2 but also CD130 and CD116, two receptors binding to IL- 6 and granulocyte-macrophage colony-stimulating factor (GM$\mathrm{CSF}$ ), respectively. When mixed with SARS-CoV-2, these NPs effectively prevented Vero cell infection and subsequent viral replication [35]. Additionally, in a mouse model of acute lung inflammation, these NPs absorbed and neutralized IL-6 and GM-CSF, leading to reduced lung injury. These innovative strategies highlight encouraging prospects for mitigating and controlling viral outbreaks (Fig. 1c).

\section{SARS-CoV-2 vaccines}

Vaccination is viewed as the best strategy to protect us all against SARS-CoV-2 transmission, post-infection complications, and the spread of COVID-19. Multiple waves of this pandemic are hitting the world, reinforcing how critical an effective vaccine is needed to stop the devastating human toll and economic damage [77]. Any vaccine must be safe for human use. Additionally, the vaccine should ideally be administered once or twice to achieve high effectiveness and induce longlasting immunity. Finally, a vaccine suitable for global use should be stable for storage and distribution [78]. In the race for SARS-CoV-2 vaccines, several approaches are being investigated, some of which meet all or many of the criteria for a suitable vaccine for global distribution. These approaches include traditional vaccines based on inactivated or live virus, virus-vectored and subunit vaccines, and radically new technologies to vaccination using RNA or DNA (Table 1) [79].

The long-awaited vaccine to end the pandemic appears to be on the horizon. Preliminary results from phase III clinical trials show great promise for mRNA vaccines. Initial data from Moderna's mRNA-1273 (NCT04470427) [80] and Pfizer/BioNTech's BNT162b2 mRNA

Table 1 Summary of SARS-CoV-2 vaccine candidates in phase 3 clinical trials

\begin{tabular}{|c|c|c|c|}
\hline Vaccine name & Vaccine type & Company and institution & Clinical trial number \\
\hline \multicolumn{4}{|c|}{ Biomaterials-related vaccines } \\
\hline BNT162 & mRNA-based vaccine & Pfizer, BioNTech & NCT04368728 \\
\hline mRNA-1273 & mRNA-based vaccine & Moderna & NCT04470427 \\
\hline \multicolumn{4}{|l|}{ Others } \\
\hline CoronaVac & Inactivated vaccine & $\begin{array}{l}\text { Sinovac } \\
\text { Butantan Institute }\end{array}$ & NCT04456595 \\
\hline Covaxin & Inactivated vaccine & $\begin{array}{l}\text { Bharat Biotech } \\
\text { National Institute of Virology }\end{array}$ & NCT04641481 \\
\hline $\begin{array}{l}\text { No name } \\
\text { announced }\end{array}$ & Inactivated vaccine & $\begin{array}{l}\text { Wuhan Institute of Biological Products } \\
\text { China National Biotec Group Company } \\
\text { Beijing Institute of Biological } \\
\text { Abu Dhabi Health Services Company } \\
\text { G42 Healthcare company }\end{array}$ & NCT04510207 \\
\hline BCG-CORONA & Live-attenuated vaccine & $\begin{array}{l}\text { University of Melbourne and Murdoch } \\
\text { Children's Research Institute } \\
\text { Radboud University Medical Center } \\
\text { Faustman Lab at Massachusetts General Hospital }\end{array}$ & NCT04328441 \\
\hline NVX-CoV2373 & Saponin-based vaccine & Novavax, Takeda & NCT04583995 \\
\hline Sputnik V & $\begin{array}{l}\text { Non-replicating viral vector } \\
\text { vaccine }\end{array}$ & $\begin{array}{l}\text { Gamaleya Research Institute } \\
\text { Dr. Reddy's Laboratories }\end{array}$ & NCT04640233 \\
\hline Gam-COVID-Vac & $\begin{array}{l}\text { Non-replicating viral vector } \\
\text { vaccine }\end{array}$ & $\begin{array}{l}\text { Gamaleya Research Institute of Epidemiology and } \\
\text { Microbiology } \\
\text { I-Pharm }\end{array}$ & NCT04564716 \\
\hline AZD1222 & $\begin{array}{l}\text { Non-replicating viral vector } \\
\text { vaccine }\end{array}$ & $\begin{array}{l}\text { University of Oxford } \\
\text { AstraZeneca } \\
\text { IQVIA } \\
\text { Covance } \\
\text { Serum Institute of India }\end{array}$ & $\begin{array}{l}\text { NCT04540393 } \\
\quad \text { (suspended) }\end{array}$ \\
\hline JNJ-78436735 & $\begin{array}{l}\text { Non-replicating viral vector } \\
\text { vaccine }\end{array}$ & Johnson \& Johnson & NCT04505722 \\
\hline $\mathrm{Ad} 5-\mathrm{nCoV}$ & Recombinant vaccine & CanSino Biologics & NCT04526990 \\
\hline
\end{tabular}



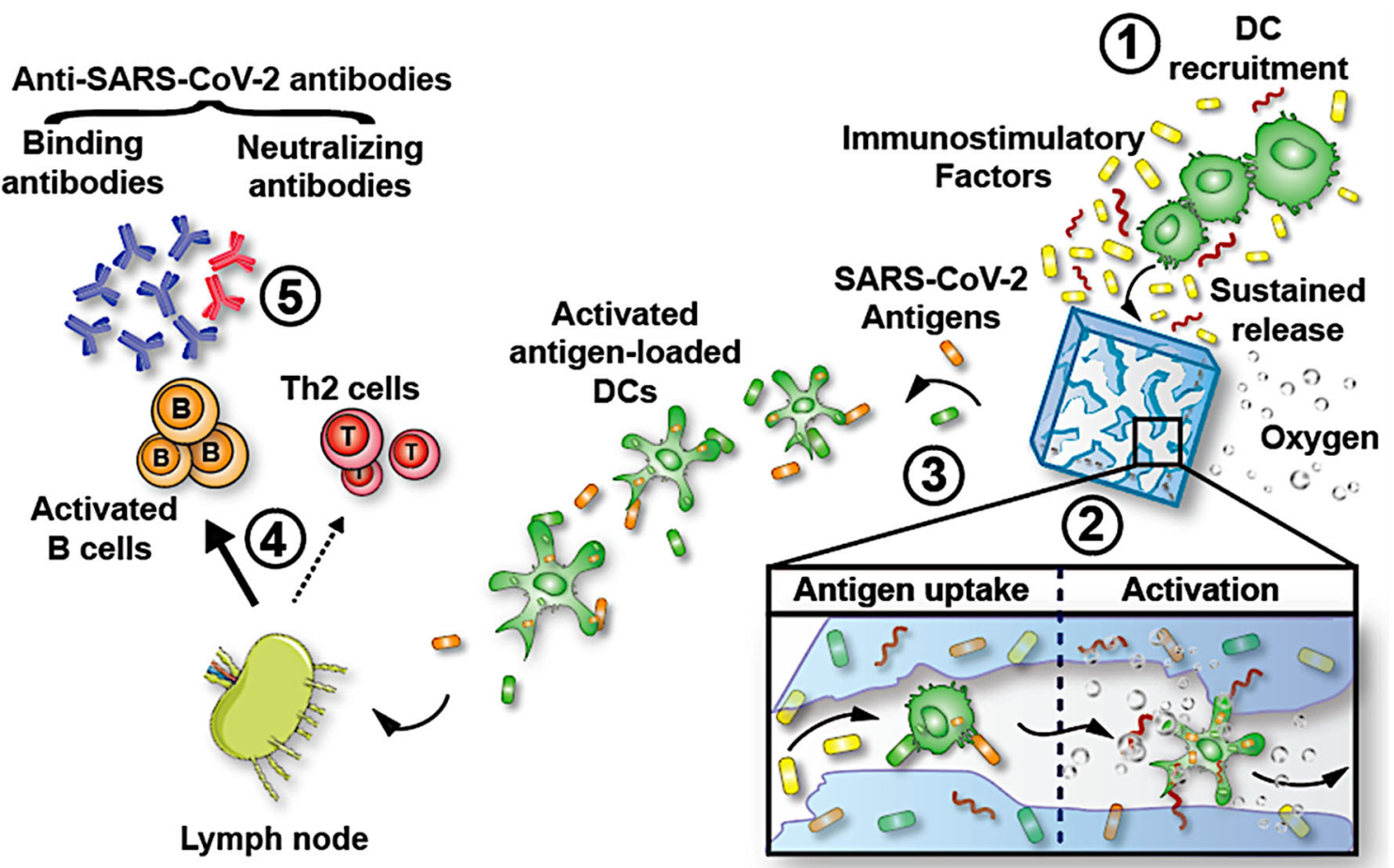

Fig. 2 Harnessing biomaterials to boost COVID-19 vaccines. Scaffolds can be designed to (1) sustain the release of immunomodulatory factors to promote dendritic cell (DC) recruitment and (2) improve local antigen uptake. Scaffolds that produce oxygen improve immune cell activation so that (3) activated, antigen-loaded DCs would migrate to draining lymph nodes to initiate the activation of antigen-specific $T$ cells and $B$ cells. Furthermore, protein antigens and adjuvants released from the scaffold

(NCT04368728) [81] vaccines are reportedly over 90\% effective in preventing COVID-19, with no safety concerns so far. These mRNA vaccines rely on lipid NPs for enhanced intracellular delivery, highlighting the pivotal role of biomaterials in achieving high vaccine efficacy [8]. However, mRNA vaccines have several limitations, such as a short shelf life, and they require very specific cold storage, complicating their logistics and distribution [82]. To overcome these shortcomings, scientists are developing thermostable vaccines using more sophisticated biomaterials. For instance, a SARS-CoV-2 mRNA vaccine encapsulated within liposomes composed of 2-distearoyl-snglycero-3-phosphocholine, cholesterol, and PEG has recently been developed. This improved version of the mRNA vaccine is stable for over 1 week at room temperature and triggers immunity against SARS-CoV-2 in mice and non-human primates [83]. Furthermore, due to the public health emergency and vaccine shortages, developing vaccines that require a minimal dose is crucial. Similarly to an effective mRNA vaccine against influenza, selfamplifying RNA vaccines have shown to provide equivalent protection but at a 64-fold lower dose [84]. This is a unique and promising approach to reduce the amount of RNA required in the formulation of SARS-CoV-2 vaccines would also drain to the lymph nodes, leading to local B cell and DC activation. (4) A subset of activated B cells would differentiate into plasma cells that produce large quantities of SARS-CoV-2-binding antibodies. (5) A fraction of these antibodies would be neutralizing antibodies and exert their inhibitory activity by abrogating binding of the virus to the ACE2 receptor (reproduced and modified with permission from Colombani et al. [93])

[85]. This new vaccine technology has recently been validated in a mouse model, leading to a Th1-biased immune response and high neutralizing antibody titers against the virus.

Another major limitation of vaccines, including mRNA vaccines, is their low immunogenicity. This is particularly concerning because the most vulnerable populations to COVID-19 are senior or immunocompromised individuals with weakened immune functions [86]. To enhance mRNA vaccine immunogenicity, one strategy is to use immunostimulatory delivery systems. For instance, diC14-amidine liposomes have been used as a delivery system for mRNA [87], and these cationic liposomes improved the immunogenicity of an aluminumbased protein vaccine by stimulating multiple signaling pathways important for immune activation [88]. Similarly, lipoaminoglycosides effectively delivered nucleic acids into cells [89] and exhibited strong immunostimulatory properties in a mouse model of breast cancer, indicating that liposomes can be used as a drug delivery carrier with adjuvant attributes [90]. Oxygen, an underrated yet powerful co-adjuvant with the potential to boost vaccine immunogenicity, is currently under investigation [91]. Our lab has recently engineered oxygengenerating cryogels $\left(\mathrm{O}_{2}\right.$-cryogels), a sophisticated and advanced macroporous hydrogel system, with the unique ability to reverse 
hypoxia-driven immunosuppression in solid tumors [92]. Prompted by the urgent need to act against this pandemic, we applied this technology to recombinant protein subunit vaccines [93] and demonstrated that combining cryogel-based biomaterials with local oxygen production synergistically reinforces both humoral and cellular immune responses in mice. Furthermore, these biomaterials impressively spiked serological levels of both binding antibodies and neutralizing antibodies against SARS-CoV-2 (Fig. 2). This technology is applicable to other vaccines, such as mRNA-based vaccines, and can potentially improve their effectiveness while reducing the vaccine dose to achieve the desired immunization outcome.

Finally, enhanced immune stimulation may be achieved by targeting the skin in lieu of the traditionally targeted muscle tissues [94]. Skin contains a large number of antigenpresenting cells and other complementary immune cells required to coordinately induce strong and long-lasting immunity [95]. Transdermal microneedle patches, an emerging technology applicable for the transcutaneous delivery of vaccines, have recently gained great interest due to their prominent properties such as low cost and excellent therapeutic efficacy in preclinical studies [79]. One example is a microneedle array (MNA)based vaccine fabricated with carboxymethyl cellulose. These MNA-based vaccines physically breach the outer layer of the skin and rapidly dissolve to deliver spike protein S1 subunit of SARS-CoV-2 within the epidermis and dermis [96]. When tested in mice, these vaccines elicited high levels of antibody titers against SARS-CoV-2 spike protein. This platform is safe and painless, thereby opening the door for the selfadministration and widespread distribution of vaccines.

\section{Conclusion}

Despite the previous SARS and MERS viral outbreaks, there was no viable strategy to stop the SARS-CoV-2 pandemic. This new virus has profoundly changed our society and created a global healthcare crisis of unprecedented magnitude. State-of-the-art biomaterials have the potential to serve as formidable tools in the fight against SARS-CoV-2. They could fast track drug discovery and screening as well as reinforce current therapeutic platforms. This perspective highlights the latest advances of biomaterials and their future directions in our collective efforts to combat the widespread infections caused by SARS-CoV-2, thus reducing the burden on the healthcare system. Promising and exciting studies are currently being investigated, and vaccines and antiviral drugs are being developed and tested at a remarkable speed. Based on the lessons taught by COVID-19, only through close and coordinated collaborations between scientists and researchers from different fields, clinicians, and industrial partners can help us tackle collectively this unprecedented pandemic and prevent future viral outbreaks.
Acknowledgements S.A.B. acknowledges support from Northeastern University (COVID-19 crisis award), NSF CAREER award (DMR 1847843), and the National Plan for Science, Technology, and Innovation (MAARIFAH), King Abdulaziz City for Science and Technology, the Kingdom of Saudi Arabia, award (15-MED5025-03). Editorial support was provided by Nancy R. Gough (BioSerendipity, LLC).

\section{Declarations}

Competing interests The authors declare no competing interests.

\section{References}

1. E. Dong, H. Du, L. Gardner, An interactive web-based dashboard to track COVID-19 in real time. Lancet Infect. Dis. 20(5), 533-534 (2020)

2. B.S. Graham, N.J. Sullivan, Emerging viral diseases from a vaccinology perspective: preparing for the next pandemic. Nat. Immunol. 19(1), 20-28 (2018)

3. M.D. Shin, S. Shukla, Y.H. Chung, V. Beiss, S.K. Chan, O.A. Ortega-Rivera, D.M. Wirth, A. Chen, M. Sack, J.K. Pokorski, N.F. Steinmetz, COVID-19 vaccine development and a potential nanomaterial path forward. Nat. Nanotechnol. 15(8), 646-655 (2020)

4. Pan, H., et al., Repurposed antiviral drugs for COVID-19 -interim WHO SOLIDARITY trial results. medRxiv, 2020: p. 2020.10.15.20209817.

5. H. Uludag, A. Pandit, L. Kuhn, Editorial: Enabling biomaterials for new biomedical technologies and clinical therapies. Front. Bioeng. Biotechnol., 8(559) (2020)

6. A. Koirala, Y.J. Joo, A. Khatami, C. Chiu, P.N. Britton, Vaccines for COVID-19: The current state of play. Paediatr. Respir. Rev. 35, 43-49 (2020)

7. L. Gu, D.J. Mooney, Biomaterials and emerging anticancer therapeutics: engineering the microenvironment. Nat. Rev. Cancer 16(1), 56-66 (2016)

8. Z. Tang, N. Kong, X. Zhang, Y. Liu, P. Hu, S. Mou, P. Liljeström, J. Shi, W. Tan, J.S. Kim, Y. Cao, R. Langer, K.W. Leong, O.C. Farokhzad, W. Tao, A materials-science perspective on tackling COVID-19. Nat Rev Mater 5(11), 847-860 (2020)

9. O. Gsib et al., Fibrin/PEG-based sequential interpenetrating polymer network for dermal tissue engineering. Biomater. Sci. 8, 7106 $7116(2020)$

10. Z.J. Rogers, M. Zeevi, S.A. Bencherif, Electroconductive hydrogels for tissue engineering: current status and future perspectives. Bioelectricity 2(3), 279-292 (2020)

11. A. Amirsadeghi et al., Vascularization strategies for skin tissue engineering. Biomater. Sci. 8(15), 4073-4094 (2020)

12. O. Gsib et al., Evaluation of fibrin-based interpenetrating polymer networks as potential biomaterials for tissue engineering. Nanomaterials 7(12), 436 (2017)

13. O. Gsib, C. Egles, S.A. Bencherif, Fibrin: An underrated biopolymer for skin tissue engineering. J. Mol. Biol. Biotech. 2, 1 (2017)

14. T. Abudula et al., Supramolecular self-assembled peptide-based vaccines. Front. Chem. 8, 598160 (2020)

15. J. Li, D.J. Mooney, Designing hydrogels for controlled drug delivery. Nat Rev Mater 1(12), 16071 (2016)

16. L.J. Eggermont, Z.J. Rogers, T. Colombani, A. Memic, S.A. Bencherif, Injectable cryogels for biomedical applications. Trends Biotechnol. 38(4), 418-431 (2020) 
17. K. Joshi Navare et al., Needle-injectable microcomposite cryogel scaffolds with antimicrobial properties. Sci. Rep. 10, 18370 (2020)

18. A. Memic et al., Effect of polymer concentration on autoclaved cryogel properties. Macromol. Mater. Eng. 305 (5):1900824 (2020)

19. Z.J. Rogers, S.A. Bencherif, Cryogels and cryogelation. Gels 5(4), 46-47 (2019)

20. P. Villard et al., Autoclavable and injectable cryogels for biomedical applications. Adv. Healthc. Mater. 8(17), 1900679 (2019)

21. M. Rezaeeyazdi, T. Colombani, A. Memic, S.A. Bencherif, Injectable hyaluronic acid-co-gelatin cryogels for tissue engineering applications. Materials 11(8), 1374 (2018)

22. M. Han et al., Extracellular matrix-based cryogels for cartilage tissue engineering. Int. J. Biol. Macromol. 93, 1410-1419 (2016)

23. T. He et al., Hyaluronic acid-based shape memory cryogel scaffolds for focal cartilage defect repair. Tissue. Eng. Part A (2021). https:// doi.org/10.1089/ten.tea.2020.0264

24. M.E. Davis, Z. Chen, D.M. Shin, Nanoparticle therapeutics: an emerging treatment modality for cancer. Nat. Rev. Drug Discov. 7(9), 771-782 (2008)

25. D. Chakhalian, R.B. Shultz, C.E. Miles, J. Kohn, Opportunities for biomaterials to address the challenges of COVID-19. J. Biomed. Mater. Res. A 108(10), 1974-1990 (2020)

26. G.U. Jeong, H. Song, G.Y. Yoon, D. Kim, Y.C. Kwon, Therapeutic strategies against COVID-19 and structural characterization of SARS-CoV-2: a review. Front. Microbiol. 11, 1723-1723 (2020)

27. Abella, B.S., et al., Efficacy and safety of hydroxychloroquine vs placebo for pre-exposure SARS-CoV-2 prophylaxis among health care workers: a randomized clinical trial. JAMA Internal Medicine, 2020.

28. D.R. Boulware, M.F. Pullen, A.S. Bangdiwala, K.A. Pastick, S.M. Lofgren, E.C. Okafor, C.P. Skipper, A.A. Nascene, M.R. Nicol, M. Abassi, N.W. Engen, M.P. Cheng, D. LaBar, S.A. Lother, L.J. MacKenzie, G. Drobot, N. Marten, R. Zarychanski, L.E. Kelly, I.S. Schwartz, E.G. McDonald, R. Rajasingham, T.C. Lee, K.H. Hullsiek, A randomized trial of hydroxychloroquine as postexposure prophylaxis for Covid-19. N. Engl. J. Med. 383(6), 517-525 (2020)

29. Dexamethasone in Hospitalized Patients with Covid-19 Preliminary Report. New England Journal of Medicine, 2020.

30. A. Shpichka et al., Engineering a model to study viral infections: bioprinting, microfluidics, and organoids to defeat coronavirus disease 2019 (COVID-19). Int. J. Bioprinting 6(4), 302-302 (2020)

31. Seyfoori, A., et al., The role of biomaterials and three dimensional (3D) in vitro tissue models in fighting against COVID-19. Biomaterials Science, 2021.

32. L. Li, Z.Y. He, X.W. Wei, Y.Q. Wei, Recent advances of biomaterials in biotherapy. Regen. Biomater 3(2), 99-105 (2016)

33. D.N. Nguyen, J.J. Green, J.M. Chan, R. Langer, D.G. Anderson, Polymeric materials for gene delivery and DNA vaccination. Adv. Mater. 21(8), 847-867 (2009)

34. E.A. Aisenbrey, W.L. Murphy, Synthetic alternatives to Matrigel. Nat Rev Mater 5(7), 539-551 (2020)

35. L. Rao, S. Xia, W. Xu, R. Tian, G. Yu, C. Gu, P. Pan, Q.F. Meng, X. Cai, D. Qu, L. Lu, Y. Xie, S. Jiang, X. Chen, Decoy nanoparticles protect against COVID-19 by concurrently adsorbing viruses and inflammatory cytokines. Proc. Natl. Acad. Sci. 117(44), 27141-27147 (2020)

36. B. Surnar, M.Z. Kamran, A.S. Shah, U. Basu, N. Kolishetti, S. Deo, D.T. Jayaweera, S. Daunert, S. Dhar, Orally administrable therapeutic synthetic nanoparticle for Zika virus. ACS Nano 13(10), 11034-11048 (2019)

37. C. Keech, G. Albert, I. Cho, A. Robertson, P. Reed, S. Neal, J.S. Plested, M. Zhu, S. Cloney-Clark, H. Zhou, G. Smith, N. Patel, M.B. Frieman, R.E. Haupt, J. Logue, M. McGrath, S. Weston, P.A. Piedra, C. Desai, K. Callahan, M. Lewis, P. Price-Abbott, N.
Formica, V. Shinde, L. Fries, J.D. Lickliter, P. Griffin, B. Wilkinson, G.M. Glenn, Phase 1-2 trial of a SARS-CoV-2 recombinant spike protein nanoparticle vaccine. N. Engl. J. Med. 383(24), 2320-2332 (2020)

38. R.S. Adcock, Y.K. Chu, J.E. Golden, D.H. Chung, Evaluation of anti-Zika virus activities of broad-spectrum antivirals and NIH clinical collection compounds using a cell-based, high-throughput screen assay. Antivir. Res. 138, 47-56 (2017)

39. L.M. Johansen et al., A screen of approved drugs and molecular probes identifies therapeutics with anti-Ebola virus activity. Sci. Transl. Med. 7(290), 290ra89-290ra89 (2015)

40. N. Osada, A. Kohara, T. Yamaji, N. Hirayama, F. Kasai, T. Sekizuka, M. Kuroda, K. Hanada, The genome landscape of the African green monkey kidney-derived Vero cell line. DNA research : an international journal for rapid publication of reports on genes and genomes 21(6), 673-683 (2014)

41. C.G.K. Ziegler et al., SARS-CoV-2 receptor ACE2 is an interferonstimulated gene in human airway epithelial cells and is detected in specific cell subsets across tissues. Cell 181(5), 1016-1035.e19 (2020)

42. M. Wang, R. Cao, L. Zhang, X. Yang, J. Liu, M. Xu, Z. Shi, Z. Hu, W. Zhong, G. Xiao, Remdesivir and chloroquine effectively inhibit the recently emerged novel coronavirus $(2019-\mathrm{nCoV})$ in vitro. Cell Res. 30(3), 269-271 (2020)

43. J. Liu, R. Cao, M. Xu, X. Wang, H. Zhang, H. Hu, Y. Li, Z. Hu, W. Zhong, M. Wang, Hydroxychloroquine, a less toxic derivative of chloroquine, is effective in inhibiting SARS-CoV-2 infection in vitro. Cell Discov. 6, 16-16 (2020)

44. D. Morniroli et al., Human sialome and coronavirus disease-2019 (COVID-19) pandemic: an understated correlation? Front. Immunol. 11(1480) (2020)

45. T. Ueland, J.C. Holter, A.R. Holten, K.E. Müller, A. Lind, G.K. Bekken, S. Dudman, P. Aukrust, A.M. Dyrhol-Riise, L. Heggelund, Distinct and early increase in circulating MMP-9 in COVID-19 patients with respiratory failure. J. Infect. 81(3), e41e43 (2020)

46. V. Wazny, A. Siau, K.X. Wu, C. Cheung, Vascular underpinning of COVID-19. Open Biol. 10(8), 200208-200208 (2020)

47. M.J. Kratochvil, A.J. Seymour, T.L. Li, S.P. Pasca, C.J. Kuo, S.C. Heilshorn, Engineered materials for organoid systems. Nat Rev Mater 4(9), 606-622 (2019)

48. R. Bhowmick, T. Derakhshan, Y. Liang, J. Ritchey, L. Liu, H. Gappa-Fahlenkamp, A three-dimensional human tissueengineered lung model to study influenza $\mathrm{A}$ infection. Tissue Eng. A 24(19-20), 1468-1480 (2018)

49. C. O'Leary et al., The development of a tissue-engineered tracheobronchial epithelial model using a bilayered collagen-hyaluronate scaffold. Biomaterials 85, 111-127 (2016)

50. M. Elbadawi, T. Efferth, Organoids of human airways to study infectivity and cytopathy of SARS-CoV-2. The Lancet. Respir Med. 8(7), e55-e56 (2020)

51. K. Takayama, $<$ em $>$ In vitro $</$ em $>$ and animal models for SARSCoV-2 research. Trends Pharmacol. Sci. 41(8), 513-517 (2020)

52. Y. Han et al., Identification of SARS-CoV-2 inhibitors using lung and colonic organoids. Nature 589, 270-275 (2021)

53. J. Zhou, C. Li, X. Liu, M.C. Chiu, X. Zhao, D. Wang, Y. Wei, A. Lee, A.J. Zhang, H. Chu, J.P. Cai, C.C.Y. Yip, I.H.Y. Chan, K.K.Y. Wong, O.T.Y. Tsang, K.H. Chan, J.F.W. Chan, K.K.W. To, H. Chen, K.Y. Yuen, Infection of bat and human intestinal organoids by SARS-CoV-2. Nat. Med. 26(7), 1077-1083 (2020)

54. V. Monteil et al., Inhibition of SARS-CoV-2 infections in engineered human tissues using clinical-grade soluble human ACE2. Cell 181(4), 905-913.e7 (2020)

55. Si, L., et al., Human organ chip-enabled pipeline to rapidly repurpose therapeutics during viral pandemics. bioRxiv (2020). https:// doi.org/10.1101/2020.04.13.039917 
56. M. Hoffmann et al., SARS-CoV-2 cell entry depends on ACE2 and TMPRSS2 and is blocked by a clinically proven protease inhibitor. Cell 181(2), 271-280.e8 (2020)

57. Y. Guo et al., SARS-CoV-2 induced intestinal responses with a biomimetic human gut-on-chip. Sci. Bull. (2020). https://doi.org/ 10.1016/j.scib.2020.11.015

58. Hassan, S., et al., Biomaterials for on-chip organ systems, in Biomaterials for organ and tissue regeneration, N.E. Vrana, H. Knopf-Marques, and J. Barthes, Editors. 2020, Woodhead Publishing. p. 669-707.

59. H. Liu, Y. Wang, K. Cui, Y. Guo, X. Zhang, J. Qin, Advances in hydrogels in organoids and organs-on-a-chip. Adv. Mater. 31(50), 1902042 (2019)

60. A. Memic et al., Latest advances in cryogel technology for biomedical applications. Adv. Ther. 2(4), 1800114 (2019)

61. M.A. Chary, A.F. Barbuto, S. Izadmehr, B.D. Hayes, M.M. Burns, COVID-19: Therapeutics and their toxicities. J. Med. Toxicol. 16(3), 284-294 (2020)

62. Pfizer-BioNTech COVID-19 Vaccine - FDA. Available at: https:// www.fda.gov/emergency-preparedness-and-response/coronavirusdisease-2019-covid-19/pfizer-biontech-covid-19-vaccine. (Accessed: 21st December 2020)

63. M.R. Casanova, R.L. Reis, A. Martins, N.M. Neves, Surface biofunctionalization to improve the efficacy of biomaterial substrates to be used in regenerative medicine. Mater. Horiz. 7(9), 2258-2275 (2020)

64. R. Croci et al., Liposomal systems as nanocarriers for the antiviral agent ivermectin. Int. J. Biomater. 2016, 8043983-8043983 (2016)

65. L. Caly, J.D. Druce, M.G. Catton, D.A. Jans, K.M. Wagstaff, The FDA-approved drug ivermectin inhibits the replication of SARSCoV-2 in vitro. Antivir. Res. 178, 104787 (2020)

66. R. Croci et al., Liposomal systems as nanocarriers for the antiviral agent ivermectin. Int. J. Biomater. 2016, 8043983 (2016)

67. J.P. Freeling et al., Long-acting three-drug combination anti-HIV nanoparticles enhance drug exposure in primate plasma and cells within lymph nodes and blood. AIDS (London, England) 28(17), 2625-2627 (2014)

68. Fan, J., et al., Connecting hydroxychloroquine in vitro antiviral activity to in vivo concentration for prediction of antiviral effect: a critical step in treating COVID-19 patients. Clinical infectious diseases: an official publication of the Infectious Diseases Society of America, 2020: p. ciaa623.

69. Tai, T.T., et al., A strategy to treat COVID-19 disease with targeted delivery of inhalable liposomal hydroxychloroquine: a pre-clinical pharmacokinetic study. Clin Transl Sci, 2020.

70. T. Lammers, A.M. Sofias, R. van der Meel, R. Schiffelers, G. Storm, F. Tacke, S. Koschmieder, T.H. Brümmendorf, F. Kiessling, J.M. Metselaar, Dexamethasone nanomedicines for COVID-19. Nat. Nanotechnol. 15(8), 622-624 (2020)

71. E.O. Gubernatorova, E.A. Gorshkova, A.I. Polinova, M.S. Drutskaya, IL-6: Relevance for immunopathology of SARSCoV-2. Cytokine Growth Factor Rev. 53, 13-24 (2020)

72. M. Uehara, X. Li, A. Sheikhi, N. Zandi, B. Walker, B. Saleh, N. Banouni, L. Jiang, F. Ordikhani, L. Dai, M. Yonar, I. Vohra, V. Kasinath, D.P. Orgill, A. Khademhosseini, N. Annabi, R. Abdi, Anti-IL-6 eluting immunomodulatory biomaterials prolong skin allograft survival. Sci. Rep. 9(1), 6535 (2019)

73. A. Zoufaly, M. Poglitsch, J.H. Aberle, W. Hoepler, T. Seitz, M. Traugott, A. Grieb, E. Pawelka, H. Laferl, C. Wenisch, S. Neuhold, D. Haider, K. Stiasny, A. Bergthaler, E. Puchhammer-Stoeckl, A. Mirazimi, N. Montserrat, H. Zhang, A.S. Slutsky, J.M. Penninger, Human recombinant soluble ACE2 in severe COVID-19. Lancet Respir. Med. 8(11), 1154-1158 (2020)

74. T.M. Abd El-Aziz, A. Al-Sabi, J.D. Stockand, Human recombinant soluble ACE2 (hrsACE2) shows promise for treating severe COVID-19. Signal Transduct Target Ther 5(1), 258 (2020)
75. Q. Zhang, A. Honko, J. Zhou, H. Gong, S.N. Downs, J.H. Vasquez, R.H. Fang, W. Gao, A. Griffiths, L. Zhang, Cellular nanosponges inhibit SARS-CoV-2 infectivity. Nano Lett. 20(7), 5570-5574 (2020)

76. S. Thamphiwatana, P. Angsantikul, T. Escajadillo, Q. Zhang, J. Olson, B.T. Luk, S. Zhang, R.H. Fang, W. Gao, V. Nizet, L. Zhang, Macrophage-like nanoparticles concurrently absorbing endotoxins and proinflammatory cytokines for sepsis management. Proc. Natl. Acad. Sci. 114(43), 11488-11493 (2017)

77. P.M. Heaton, The Covid-19 vaccine-development multiverse. N. Engl. J. Med. 383(20), 1986-1988 (2020)

78. D.R. Burton, Antibodies, viruses and vaccines. Nat. Rev. Immunol. 2(9), 706-713 (2002)

79. F. Krammer, SARS-CoV-2 vaccines in development. Nature 586(7830), 516-527 (2020)

80. L.A. Jackson et al., mRNA-1273 Study Group, An mRNA vaccine against SARS-CoV-2 - preliminary report. N. Engl. J. Med. 383(20), 1920-1931 (2020)

81. M.J. Mulligan et al., Phase I/II study of COVID-19 RNA vaccine BNT162b1 in adults. Nature 586(7830), 589-593 (2020)

82. N. Pardi, M.J. Hogan, F.W. Porter, D. Weissman, mRNA vaccines - a new era in vaccinology. Nat. Rev. Drug Discov. 17(4), 261279 (2018)

83. N.N. Zhang et al., A thermostable mRNA vaccine against COVID19. Cell 182(5), 1271-1283.e16 (2020)

84. A.B. Vogel, L. Lambert, E. Kinnear, D. Busse, S. Erbar, K.C. Reuter, L. Wicke, M. Perkovic, T. Beissert, H. Haas, S.T. Reece, U. Sahin, J.S. Tregoning, Self-amplifying RNA vaccines give equivalent protection against influenza to mRNA vaccines but at much lower doses. Mol. Ther. 26(2), 446-455 (2018)

85. P.F. McKay, K. Hu, A.K. Blakney, K. Samnuan, J.C. Brown, R. Penn, J. Zhou, C.R. Bouton, P. Rogers, K. Polra, P.J.C. Lin, C. Barbosa, Y.K. Tam, W.S. Barclay, R.J. Shattock, Self-amplifying RNA SARS-CoV2 lipid nanoparticle vaccine candidate induces high neutralizing antibody titers in mice. Nat. Commun. 11(1), 3523 (2020)

86. C.A. DiazGranados, A.J. Dunning, M. Kimmel, D. Kirby, J. Treanor, A. Collins, R. Pollak, J. Christoff, J. Earl, V. Landolfi, E. Martin, S. Gurunathan, R. Nathan, D.P. Greenberg, N.G. Tornieporth, M.D. Decker, H.K. Talbot, Efficacy of high-dose versus standard-dose influenza vaccine in older adults. N. Engl. J. Med. 371(7), 635-645 (2014)

87. A. El Ouahabi et al., Double long-chain amidine liposomemediated self replicating RNA transfection. FEBS Lett. 380(1-2), 108-112 (1996)

88. A. Wilmar, C. Lonez, M. Vermeersch, M. Andrianne, D. PérezMorga, J.M. Ruysschaert, M. Vandenbranden, O. Leo, S.T. Temmerman, The cationic lipid, diC14 amidine, extends the adjuvant properties of aluminum salts through a TLR-4- and caspase-1independent mechanism. Vaccine 30(2), 414-424 (2012)

89. D. Habrant, P. Peuziat, T. Colombani, L. Dallet, J. Gehin, E. Goudeau, B. Evrard, O. Lambert, T. Haudebourg, B. Pitard, Design of ionizable lipids to overcome the limiting step of endosomal escape: application in the intracellular delivery of mRNA, DNA, and siRNA. J. Med. Chem. 59(7), 3046-3062 (2016)

90. T. Colombani, T. Haudebourg, M. Decossas, O. Lambert, G. Ada da Silva, F. Altare, B. Pitard, Lipidic aminoglycoside derivatives: a new class of immunomodulators inducing a potent innate immune stimulation. Advanced Science 6(16), 1900288 (2019)

91. S.M. Hatfield, M. Sitkovsky, Oxygenation to improve cancer vaccines, adoptive cell transfer and blockade of immunological negative regulators. Oncoimmunology 4(12), e1052934-e1052934 (2015)

92. Colombani, T., et al., Oxygen-generating cryogels restore T cellmediated cytotoxicity in hypoxic tumors. bioRxiv (2020). https:// doi.org/10.1101/2020.10.08.329805

93. T. Colombani, L.J. Eggermont, Z.J. Rogers, L. McKay, L. Avena, R. Johnson, N. Storm, A. Griffiths, S.A. Bencherif. Biomaterials 
and oxygen join forces to shape the immune response and boost SARS-CoV-2 vaccines. Research Square (2020). https://doi.org/10. 21203/rs.3.rs-102055/v1

94. A.V. Boopathy, A. Mandal, D.W. Kulp, S. Menis, N.R. Bennett, H.C. Watkins, W. Wang, J.T. Martin, N.T. Thai, Y. He, W.R. Schief, P.T. Hammond, D.J. Irvine, Enhancing humoral immunity via sustained-release implantable microneedle patch vaccination. Proc. Natl. Acad. Sci. 116(33), 16473-16478 (2019)
95. K. Kabashima, T. Honda, F. Ginhoux, G. Egawa, The immunological anatomy of the skin. Nat. Rev. Immunol. 19(1), 19-30 (2019)

96. E. Kim, G. Erdos, S. Huang, T.W. Kenniston, S.C. Balmert, C.D. Carey, V.S. Raj, M.W. Epperly, W.B. Klimstra, B.L. Haagmans, E. Korkmaz, L.D. Falo Jr., A. Gambotto, Microneedle array delivered recombinant coronavirus vaccines: immunogenicity and rapid translational development. EBioMedicine 55, 102743 (2020) 